\section{Visual function assessment in simulated real-life situations in patients with age-related macular degeneration compared to normal subjects}

\author{
Abstract \\ Purpose To evaluate visual function \\ variations in eyes with age-related macular \\ degeneration (AMD) compared to normal \\ eyes under different light/contrast conditions \\ using a time-dependent visual acuity testing \\ instrument, the Central Vision Analyzer \\ (CVA). \\ Methods Overall, 37 AMD eyes and 35 \\ normal eyes were consecutively tested with \\ the CVA after assessing best-corrected visual \\ acuity (BCVA) using ETDRS charts. The CVA \\ established visual thresholds for three \\ mesopic environments (M1 (high contrast), \\ M2 (medium contrast), and M3 (low \\ contrast)) and three backlight-glare \\ environments (G1 (high contrast, equivalent \\ to ETDRS), G2 (medium contrast), and G3 \\ (low contrast)) under timed conditions. \\ Vision drop across environments was \\ calculated, and repeatability of visual scores \\ was determined. \\ Results BCVA significantly reduced with \\ decreasing contrast in all eyes. M1 scores for \\ BCVA were greater than M2 and M3 \\ $(P<0.001) ; \mathrm{G} 1$ scores were greater than G2 \\ and G3 $(P<0.01)$. BCVA dropped more in \\ AMD eyes than in normal eyes between M1 \\ and M2 $(P=0.002)$ and between M1 and M3 \\ $(P=0.003)$. In AMD eyes, BCVA was better \\ using ETDRS charts compared to G1 \\ $(P<0.001)$. The drop in visual function \\ between ETDRS and G1 was greater in AMD \\ eyes compared to normal eyes $(P=0.004)$. \\ Standard deviations of test-retest ranged \\ from 0.100 to $0.139 \log$ MAR.
}

G Barteselli1,2, ML Gomez ${ }^{1}$, AL Doede ${ }^{1}$, J Chhablani ${ }^{3}$, W Gutstein ${ }^{4}$, D-U Bartsch ${ }^{1}$, L Dustin $^{5}$, SP Azen ${ }^{5}$ and WR Freeman ${ }^{1}$

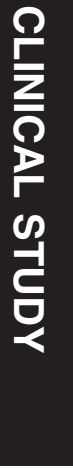

${ }^{1}$ Department of Ophthalmology, Jacobs Retina Center at Shiley Eye Center, University of California, San Diego, La Jolla, CA, USA

Conclusion The CVA allowed analysis of the visual complaints that AMD patients experience with different lighting/contrast time-dependent conditions. BCVA changed significantly under different lighting/contrast conditions in all eyes, however, AMD eyes were more affected by contrast reduction than normal eyes. In AMD eyes, timed conditions using the CVA led to worse BCVA compared to non-timed ETDRS charts. Eye (2014) 28, 1231-1238; doi:10.1038/eye.2014.189; published online 1 August 2014

\section{Introduction}

Age-related macular degeneration (AMD) is the leading cause of irreversible blindness in the developed world, and causes considerable morbidity despite recent advances in treatment. ${ }^{1}$ Patients with AMD now may be treated using a variety of preventive methods, ${ }^{2}$ and anti-vascular endothelial growth factor (VEGF) therapy for choroidal neovascularization has converted wet AMD from a disease often resulting in vision of $20 / 200$ or worse to one where patients may enjoy vision of $20 / 40 .{ }^{3}$ However, despite the visual outcome may be better than it was before the anti-VEGF therapy, patients with treated wet AMD often complain about difficulties performing tasks of daily living.

The Snellen chart test is the most commonly used chart to evaluate vision in clinical setting. More recently, the ETDRS chart has become the gold-standard test for best-corrected visual
2Ophthalmological Unit, Department of Clinical Sciences and Community Health, Ca' Granda Foundation-Ospedale Maggiore Policlinico, University of Milan, Milan, Italy

${ }^{3} \mathrm{~L} V$ Prasad Eye Institute, Hyderabad, India

${ }^{4}$ Salus University, Elkins Park, PA, USA

${ }^{5}$ Department of Preventive Medicine, Keck School of Medicine, University of Southern California, Los Angeles, CA, USA

Correspondence: WR Freeman, Department of Ophthalmology, Jacobs Retina Center at Shiley Eye Center, University of California, San Diego, 0946, 9415 Campus Point Drive, La Jolla, CA 92037. USA

Tel: + 1858534 3513; Fax: + 18585347985

E-mail: freeman@ eyecenter.ucsd.edu

Received: 9 February 2014 Accepted in revised form: 3 July 2014

Published online: 1 August 2014 
acuity (BCVA) in clinical trials evaluating eyes with macular disease. ${ }^{4}$ However, these are non-timed tests and thus may not reflect real-life situations such as driving, where the patient has only a limited amount of time to recognize objects and read signs and make important visual-based decisions. This is important in patients with AMD because many of the vision tasks that they complain about involve visual difficulties in time-dependent experiences including reading, driving, and recognizing faces in fluid social situations. ${ }^{5}$ In addition, well-known issues of scoring the Snellen chart test and the ETDRS test are also present: means of calculating BCVA scores may vary between examiners, as patients are often able to read only some letters but from multiple lines. ${ }^{6,7}$ Moreover, these chart tests use high-contrast black letters against an unchanging white background of one luminance in the moderate photopic environment of the examination office. For these reasons, other investigators have suggested that these chart tests are not a useful determination of vision in other contrast or luminance environments, and those measurements are poorly correlated with daily living activities $^{8,9}$ that require the patient to assess visual stimuli in multiple lighting, contrast, or glare conditions against a varied background color or luminance. ${ }^{7}$ Also, many common daily activities require an individual to react to a visual stimulus with limited time or information. 7,10

Testing of multiple aspects of patients' visual function (such as contrast sensitivity, luminance adaption, color perception, and reading speed) currently must be performed with separate tests and scoring methods. Recently, the Central Vision Analyzer (CVA; Visoptics, LLC, Mechanicsburg, PA, USA) has been introduced and approved by the Food and Drug Administration as a new interactive computer program to perform a comprehensive analysis of the central visual acuity under conditions that simulate environments of real-world activities that are often reported as visually stressful, such as those encountered under mesopic, lighting, or glare photopic conditions. ${ }^{11-13}$ It also simulates the fixation times that are required for those activities. Unlike the ETDRS chart test, the CVA presents tumbled Landolt $\mathrm{C}$ and thresholds for the smallest $\mathrm{C}$ for which the patient responds the correct position under the luminance, contrast and presentation times of the activity, watching and examining reaction times, and thus avoiding letter confusion with the consistent use of one symbol. ${ }^{10}$

The purpose of the present study was to evaluate how visual function varies in patients with AMD compared with normal subjects tested with this novel computerized test of time-dependent contrast sensitivity under conditions that simulate real-world environments.

\section{Materials and methods}

\section{Subjects}

We recruited 35 normal eyes and 37 eyes with an ophthalmoscopic diagnosis of wet or dry AMD, confirmed with retinal imaging, from 53 consecutive patients. Eyes with dry AMD had drusen, pigment changes, and/or drusenoid pigment epithelial detachment; all patients with wet AMD were under treatment with an anti-VEGF agent. Eyes with a concomitant ocular abnormality (eg, corneal opacity, cataract, or glaucoma) were not tested. Prior to CVA testing, all participants underwent refraction and BCVA assessment using a standard ETDRS chart. A full optical correction was given to each patient, if necessary. Written informed consent for non-invasive diagnostic procedure was obtained from each subject before examination. The study was approved by the Institutional Review Board at Shiley Eye Center at the University of California, San Diego (UCSD) and was conducted in adherence to the tenets of the Declaration of Helsinki. All applicable institutional and governmental regulations concerning the ethical use of human volunteers were followed during this research.

\section{Visual function testing}

Automated visual acuity testing was performed using the CVA. Participants were placed in a dark room of luminance $<5 \mathrm{Cd} / \mathrm{m}^{2}$, sitting at a distance of $5 \mathrm{~m}$ from the testing 20-inch LED monitor, as calculated by the combined distance from the participant to the mirror and from the mirror to the testing monitor. ${ }^{12}$ First, an instruction video with a practice test was given: participants were shown a fixation cross on the monitor and asked to indicate the orientation of the tumbled Landolt $\mathrm{C}$ by pressing the corresponding arrow button 'right,' 'left,' 'up', or 'down' on a keypad on their lap. If they pressed the correct button, the response would be counted as correct. Any other button pressing, including no pressing within the maximum time period (up to $4 \mathrm{~s}$ ), would be counted as incorrect. After the instruction video, patients performed the CVA testing twice using a Landolt C presentation of $900 \mathrm{~ms}$. Our initial experience suggested that the $900-\mathrm{ms}$ stimulus duration is well tolerated and fast enough to simulate a real-world, timedependent situation, while patients expressed concern when tested with shorter presentations. Participants were allowed $15 \mathrm{~min}$ of rest in a dimly lit room between the two sessions.

As previously described by our group, ${ }^{13}$ the CVA thresholds acuity under three mesopic and three photopic (backlight glare) environments in sequential manner, automatically changing contrast and luminance 
levels. Contrasts and luminance levels of the six modules are presented in Table 1. Specifically, they were 99\% Michelson contrast (MC) module with white letters against a $3 \mathrm{Cd} / \mathrm{m}^{2}$ background ('M1' module), followed by lower-contrast mesopic module of $64 \%$ MC against a $3 \mathrm{Cd} / \mathrm{m}^{2}$ background ('M2' module, simulating an environment similar to a dimly lit restaurant), and then an even lower-contrast module of $43 \% \mathrm{MC}$ against a $3 \mathrm{Cd} / \mathrm{m}^{2}$ background ('M3' module, simulating visual activities encountered while driving at dusk). Immediately following the mesopic conditions, backlight-glare conditions were then tested in the following order: $10 \% \mathrm{MC}$ module with black letters against a $200 \mathrm{Cd} / \mathrm{m}^{2}$ background ('G2' module, simulating playing golf or tennis outside with the sun over head), followed by an even lower-contrast module of $8 \% \mathrm{MC}$ against a $200 \mathrm{Cd} / \mathrm{m}^{2}$ background ('G3' module that simulates playing golf or tennis outside under the sun $15^{\circ}$ off-axis), and finally ending with the 'G1' module, a full-contrast module of black letters presented at $99 \% \mathrm{MC}$ against a $200 \mathrm{Cd} / \mathrm{m}^{2}$ background. During the test, the CVA software automatically adjusts the size of the $\mathrm{C}$ depending upon the responses in a reversing staircase with progressively diminishing steps $(6: 4: 2: 1$ of logMAR 0.05 steps) until a threshold is reached, determined by two correct responses at the lowest $\log$ MAR 0.05 presentation with two incorrect responses at one $\log$ MAR 0.05 step smaller. At the end of the test, results were automatically presented to the examiner in a report as six separate visual acuity threshold scores, one for each of the simulated lighting and contrast conditions.

\section{Validation of the CVA}

The validation study of the CVA is currently under review and therefore is not part of the present study. Validation data presented at the Association for Research in Vision and Ophthalmology meetings (Gutstein W et al IOVS 2006, 47, ARVO E-Abstract 92; Sinclair SH et al IOVS 2009, 50, ARVO E-Abstract 3977; Gutstein W et al IOVS 2009, 50, ARVO E-Abstract 3212; and Gutstein Wet al IOVS
2012, 53, ARVO E-Abstract 3056), in brief, demonstrated 0.50 to 0.96 correlation with ETDRS charts presenting the same contrast and luminance with Bland and Altman statistics that demonstrate similar reproducibility and comparative visual acuities as acuity measurements with charts. One may question the ability of the CVA to measure sun glare environments on an LCD or LED monitor in a darkened room. The CVA reduces the contrast of the visualized targets against the background according to the research of $\operatorname{Vos}^{14}$ that predicted the resultant decrease in contrast for a 30-year-old individual visualizing the targets with the sun off-axis. This is defined by the off-axis distance of glare source and the ratio of the luminance of that source compared with the average luminance of the discriminated targets, not the absolute luminance (whether the source is the sun in daylight glare environments or headlight glare in mesopic environments).

For each activity, vision tasks were identified (eg, facial recognition and menu reading, among others for the activity restaurant dining) with their respective discriminated targets and backgrounds that were measured from electronic RAW images taken of each activity. Each element and the respective background represent point sources in the International Commission on Illumination (CIE) color space defined by their $L \times a \times b \times$ values (where ' $L$ ' represents luminance, ' $a$ ' represents the color hue, and ' $b$ ' represents the saturation of the color from white light occupying the center of the space). The color contrast between the two points is then represented as $\Delta E / L$, the calculated Euclidean distance between the two points in the CIE color space. ${ }^{15,16}$ To define the black and white contrast of the Landolt $C$ presented against the pedestal and background, the $\Delta E / L$ contrasts were converted to the equivalent MCs for the averages of the most important vision tasks defined by participants of the activity (in each case not $<10$ persons), and then examined to define those that required the finest resolution among the higher ranked.

Because the system does not utilize anti-aliasing, careful consideration if given of the monitor to subject's

Table 1 Central Vision Analyzer modules' characteristics

\begin{tabular}{|c|c|c|c|c|c|}
\hline $\begin{array}{l}\text { CVA } \\
\text { modules }\end{array}$ & $\begin{array}{l}\text { Contrast } \\
(\mathrm{MC}, \%)\end{array}$ & $\begin{array}{l}\text { Landolt C } \\
\text { color }\end{array}$ & $\begin{array}{c}\text { Landolt } C \\
\text { luminance }\left(C d / m^{2}\right)\end{array}$ & $\begin{array}{c}\text { Background } \\
\text { luminance }\left(\mathrm{Cd} / \mathrm{m}^{2}\right)\end{array}$ & Test simulates \\
\hline M1 & 99 & White & 220 & 3 & Mesopic_full contrast \\
\hline M2 & 64 & Gray & 4.8 & 3 & Mesopic_-dim restaurant \\
\hline M3 & 43 & Gray & 8.4 & 3 & Mesopic—driving at dusk \\
\hline G1 & 99 & Black & 1.6 & 200 & Glare-full contrast \\
\hline G2 & 10 & Gray & 180 & 200 & Glare-playing sports with sun over head \\
\hline G3 & 8 & Gray & 186 & 200 & Glare-playing sports under the sun $15^{\circ}$ off-axis \\
\hline
\end{tabular}

Abbreviations: CVA, Central Vision Analyzer; $\mathrm{Cd} / \mathrm{m}^{2}$, Candelas per meter squared; MC, Michelson contrast. 
face viewing distances such that, given the pixel step sizes, the gap openings of the smallest Landolt $\mathrm{C}$ must represent no greater than a $10 \%$ variance from the correct size. The test monitor chart background luminance and contrast of the presented figures were controlled by calibration measurements that were repeated at required monthly intervals using the Pantone Huey colorimeter (Pantone LLC, Carlstadt, NJ, USA). In addition, the colorimeter is set facing toward the mirror in order to measure and control the luminance of the wall (and hence room luminance viewed) by the patient during the testing.

\section{Statistical analysis}

Statistical analyses were performed using SAS statistical software version 9.2 (SAS Inc., Cary, NC, USA). Main outcome measures in this study were the threshold logMAR BCVA scores for each CVA module. The repeatability of the BCVA scores was evaluated by the standard deviation (SD) of repeated measurements (test-retest) described by Bland and Altman. ${ }^{17}$

Generalized estimating equations, adjusted for age, were used to test for the overall significance of BCVA measurements across categories. Bonferroni-adjusted $P$-values were used for multiple pairwise comparisons. Pearson's correlation analysis was completed using subject-averaged logMAR BCVA measurements. A $P$-value $<0.05$ was considered to be statistically significant.

\section{Results}

The mean age of the 53 subjects (72 eyes) enrolled in the study was 39 years for normal patients (range 20-69 years, 35 eyes) and 79 years for AMD patients (range 46-83 years, 37 eyes; $P<0.001$ ). The median BCVA was $20 / 20$ and 20/32 for normal and pathological groups, respectively. The average duration for the CVA testing was $2.1 \mathrm{~min}$ per eye in normal eyes (range 1.5-3.0 min), and $3.5 \mathrm{~min}$ per eye in eyes with AMD (range

2.0-4.0 $\mathrm{min}$ ).

Both in normal subjects and in AMD patients, visual function significantly reduced with decreasing contrast and luminance in all mesopic and glare environments (Table 2). M1 scores (high-contrast environment) were significantly better than M2 and M3 (low-contrast environments; both $P<0.001$ ). Similarly, G1 scores (highcontrast environment) were significantly better than G2 and G3 (low-contrast environments; both $P<0.01$ ). In AMD eyes, the visual function dropped to a greater extent than seen in normal eyes with decreasing contrast and luminance in mesopic environments $(P=0.002$ between M1 and M2, and $P=0.003$ between M1 and M3; Table 2 and Figure 1). In addition, the drop between M1 and M3 was significantly greater in eyes with wet AMD compared to eyes with dry $\operatorname{AMD}(P=0.003)$; no different visual drops were found in glare environments between wet and dry AMD eyes. The greater drop of visual function for AMD patients compared to normal subjects was not found in glare environments $(P=0.957$ between $\mathrm{G} 1$ and

Table 2 Comparison between visual acuity results for each module by disease group

\begin{tabular}{|c|c|c|c|c|}
\hline \multirow[t]{2}{*}{ Eyes } & \multicolumn{2}{|c|}{$\log M A R($ mean $\pm S E)$} & \multirow[t]{2}{*}{ Difference } & \multirow[t]{2}{*}{ P-value ${ }^{\mathrm{a}}$} \\
\hline & M2 & M1 & & \\
\hline AMD & $0.763 \pm 0.055$ & $0.346 \pm 0.037$ & $0.417 \pm 0.062$ & $<0.001$ \\
\hline Normal & $0.169 \pm 0.047$ & $-0.015 \pm 0.058$ & $0.185 \pm 0.036$ & $<0.001$ \\
\hline \multirow[t]{2}{*}{$P$-value } & & & 0.002 & \\
\hline & M3 & M1 & & \\
\hline AMD & $0.908 \pm 0.071$ & $0.346 \pm 0.037$ & $0.562 \pm 0.076$ & $<0.001$ \\
\hline Normal & $0.261 \pm 0.050$ & $-0.015 \pm 0.058$ & $0.277 \pm 0.045$ & $<0.001$ \\
\hline \multirow[t]{2}{*}{$P$-value } & & & 0.003 & \\
\hline & $\mathrm{G} 2$ & G1 & & \\
\hline AMD & $0.560 \pm 0.056$ & $0.417 \pm 0.034$ & $0.143 \pm 0.049$ & 0.007 \\
\hline Normal & $0.168 \pm 0.077$ & $-0.0004 \pm 0.050$ & $0.168 \pm 0.050$ & 0.003 \\
\hline \multirow[t]{2}{*}{$P$-value } & & & 0.957 & \\
\hline & G3 & G1 & & \\
\hline AMD & $0.565 \pm 0.056$ & $0.417 \pm 0.034$ & $0.148 \pm 0.048$ & 0.004 \\
\hline Normal & $0.187 \pm 0.080$ & $-0.0004 \pm 0.050$ & $0.187 \pm 0.062$ & 0.007 \\
\hline \multirow[t]{2}{*}{$P$-value } & & & 0.791 & \\
\hline & ETDRS & G1 & & \\
\hline AMD & $0.184 \pm 0.028$ & $0.417 \pm 0.034$ & $-0.233 \pm 0.033$ & $<0.001$ \\
\hline Normal & $-0.076 \pm 0.025$ & $-0.0004 \pm 0.050$ & $-0.076 \pm 0.045$ & 0.100 \\
\hline$P$-value & & & 0.004 & \\
\hline
\end{tabular}

Abbreviations: AMD, age-related macular degeneration; SE, standard error. The bold values are $P$-values, obtained from the comparison between the $\log$ MAR differences among AMD and normal eyes.

${ }^{a}$ Using paired $t$-test. 


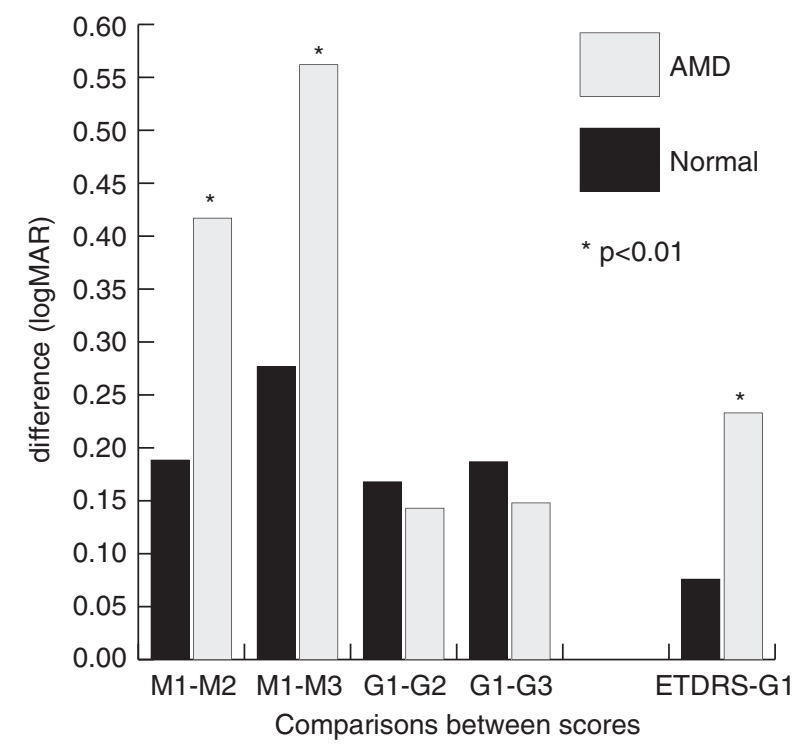

Figure 1 Clustered column graph showing comparisons between visual acuity drops for normal eyes and eyes with age-related macular degeneration (AMD) while changing lighting/contrast conditions using the Central Vision Analyzer. The visual function dropped more in AMD eyes than normal eyes while decreasing contrast and Landolt $C$ luminance in mesopic environments $(P=0.002$ between $\mathrm{M} 1$ and $\mathrm{M} 2$, and $P=0.003$ between M1 and M3). This greater drop for AMD patients compared to normal patients was not found in glare environments ( $P=0.957$ between $\mathrm{G} 1$ and $\mathrm{G} 2$, and $P=0.791$ between G1 and G3). The drop in visual function between ETDRS and G1 was significantly greater in AMD eyes compared to normal eyes $(P=0.004)$.

G2, and $P=0.791$ between G1 and G3; Table 2 and Figure 1).

We also compared results of the logMAR BCVA of the G1 module (the closest one to lighting of the ETDRS chart-high-contrast black type over a bright white background) to the logMAR ETDRS BCVA (Table 2). In AMD eyes, we found that BCVA score was significantly different between ETDRS and G1 $(P<0.001)$; more specifically, the ETDRS score was better than the G1 score. On the contrary, ETDRS and G1 scores for BCVA did not differ for normal patients $(P=0.100)$. The drop in visual function between ETDRS and G1 was significantly greater in AMD eyes compared to normal eyes $(P=0.004$; Table 2 and Figure 1).

Table 3 summarizes results of the test-retest coefficients of repeatability (equal to $1.96 \times$ standard error sigma) for each of the CVA modules. The SDs of test-retest ranged from 0.100 to $0.129 \log$ MAR in normal eyes, from 0.118 to $0.139 \log$ MAR in eyes with AMD, and from 0.100 to 0.132 in the entire sample. Independent $t$-test demonstrated no significant difference in repeatability scores between normal and AMD eyes. Bland-Altman plots for visual function measurements in each CVA environment are shown in Figure 2, both for
Table 3 Results of repeatability of visual acuity scores using Central Vision Analyzer (logMAR units)

\begin{tabular}{|c|c|c|c|c|c|c|}
\hline & \multicolumn{2}{|c|}{$\begin{array}{l}\text { Normal eyes } \\
(\mathrm{N}=35)\end{array}$} & \multicolumn{2}{|c|}{$\begin{array}{c}\text { AMD eyes } \\
(\mathrm{N}=37)\end{array}$} & \multicolumn{2}{|c|}{$\begin{array}{l}\text { All eyes } \\
(\mathrm{N}=72)\end{array}$} \\
\hline & $\begin{array}{c}S D \\
\text { test-retest }\end{array}$ & $\begin{array}{l}95 \% \\
L O A\end{array}$ & $\begin{array}{c}S D \\
\text { test-retest }\end{array}$ & $\begin{array}{l}95 \% \\
L O A\end{array}$ & $\begin{array}{c}S D \\
\text { test-retest }\end{array}$ & $\begin{array}{l}95 \% \\
L O A\end{array}$ \\
\hline M1 & 0.125 & 0.244 & 0.139 & 0.273 & 0.131 & 0.257 \\
\hline M2 & 0.100 & 0.197 & 0.121 & 0.238 & 0.110 & 0.215 \\
\hline M3 & 0.116 & 0.228 & 0.118 & 0.231 & 0.117 & 0.230 \\
\hline G1 & 0.129 & 0.253 & 0.136 & 0.267 & 0.132 & 0.259 \\
\hline G2 & 0.110 & 0.215 & 0.121 & 0.237 & 0.114 & 0.224 \\
\hline G3 & 0.108 & 0.212 & 0.122 & 0.239 & 0.114 & 0.224 \\
\hline
\end{tabular}

Abbreviations: AMD, age-related macular pathology; LOA, limits of agreement; SD, standard deviation.

normal eyes and AMD eyes. Scrutiny of Bland-Altman charts did not demonstrate any untoward relationship between agreement and magnitude of any measures, with random scatter above and below zero and no significant bias.

\section{Discussion}

In the present study, we used the CVA to evaluate visual function in different mesopic- and backlight-glare environments in eyes with AMD compared to normal eyes, using time-dependent settings to simulate real-life conditions. BCVA scores reduced significantly with decreasing contrast and luminance conditions in both normal and AMD eyes. However, the visual function in AMD eyes was more affected than in normal eyes by the decreasing contrast in mesopic conditions; that is, the magnitude of vision drop was larger for AMD eyes than for normal eyes. In addition, we found that ETDRS scores and G1 scores for BCVA were equal only for normal eyes, while the ETDRS charts measured a better acuity compared to the G1 module (timed test) for AMD eyes. This means that, under timed conditions, AMD eyes (and not normals) have significantly lower acuity than expected. As discussed further below, CVA testing was generally a repeatable method, with repeatability within 0.10 and $0.14 \log$ MAR for both normal and AMD eyes.

In an era of important technological treatment advances in AMD and other ophthalmic disorders, it is important to understand the real-world effects on vision of such interventions. For example, a patient with treated wet AMD may achieve a BCVA of 20/40 but the quality of that vision may not be good in everyday situations where speed-related vision tasks at varying contrast or background illumination are common. The Snellen chart test and the ETDRS chart test are the current standard methods for testing BCVA in clinical and research settings, ${ }^{4}$ and Pelli-Robson charts are one of the most 



Figure 2 Bland-Altman plots for visual function measures for normal eyes (a-f) and for eyes with age-related macular degeneration (g-1) in the six modules of the Central Vision Analyzer. (a,g) 'M1' module (full-contrast module of white letters presented at $99 \%$ contrast against a $3 \mathrm{Cd} / \mathrm{m}^{2}$ background). (b,h): 'M2' module (lower-contrast module of $64 \%$ contrast against a $3 \mathrm{Cd} / \mathrm{m}^{2}$ background, simulating an environment similar to a dimly lit restaurant). (c,i) ' $\mathrm{M} 3$ ' module and (lower-contrast module of $43 \%$ contrast against a $3 \mathrm{Cd} / \mathrm{m}^{2}$ background, simulating an environment similar to driving at dusk). (d,j) 'G1' module (full-contrast module of black letters presented at $99 \%$ contrast against a $200 \mathrm{Cd} / \mathrm{m}^{2}$ background). (e,k) ' $\mathrm{G} 2^{\prime}$ module (lower contract module of $10 \%$ contrast against a $200 \mathrm{Cd} / \mathrm{m}^{2}$ background, simulating playing golf or tennis outside with the sun over head). (f,l) 'G3' module (lower-contrast module of $8 \%$ contrast against a $200 \mathrm{Cd} / \mathrm{m}^{2}$ background, simulating an environment similar to playing golf or tennis outside under the sun $15^{\circ}$ off-axis).

common methods for testing contrast sensitivity. However, these tests allow the patient to take an indefinite amount of time to recognize the target; this is not reproducing real-world experiences, which are timedependent. The Pelli-Robson contrast chart requires different test cards than the ETDRS, has a different illumination, and tests only contrast at large spatial frequencies. On the contrary, the CVA tests contrast at both high and low spatial frequencies (ie, high and low visual acuity). Moreover, the Snellen testing is less accurate than ETDRS in patients with AMD probably because of its lower contrast and brightness, resulting in lower acuity measurements than ETDRS. ${ }^{18}$ With the use of the timed CVA testing we demonstrated that lighting and contrast variations significantly reduce the visual performance for both normal and AMD subjects. 
However, contrast reduction affects AMD patients more adversely than normal subjects; in addition, eyes with wet AMD experience more trouble in low-contrast

environments than dry AMD eyes. This is consistent with results of previous studies that reported delayed light adaptation and reduced contrast sensitivity in patients with AMD. ${ }^{19,20}$ We did not find differences in drops of visual function between normal and AMD eyes in backlight-glare environments. The presence of a smaller pupil size in elderly population may account for the lack of effect of backlight glare, and therefore explain this finding.

The luminance and contrast of the CVA's G1 module is most similar to that of the ETDRS chart test (eg, highcontrast black type over a bright white background). We demonstrated that in eyes with AMD, testing BCVA with conventional non-timed ETDRS charts lead to better visual scores compared to the timed G1 testing; this finding is likely due to the time-dependent nature of the automated CVA testing. It is known that patients with macular pathology have a slower reading speed than normal patients ${ }^{21}$ and thus may fail to recognize letters if projected only for a short period of time. However, we emphasize that in real-life situations one does not have all the time he wants to read something, such as reading a road sign while driving, or reading a sign on a moving object. Therefore, we believe that a time-dependent test for BCVA, such as the CVA, may reproduce real-life situations better than a static ETDRS chart.

We also tested the repeatability of the CVA testing. The test-retest coefficients of repeatability of the CVA were found to be within 0.10 and $0.14 \log$ MAR for both normal and AMD eyes, without significant differences between the two groups. At first glance, the coefficients of repeatability of the CVA appear to be slightly larger than those that are reported for reading $\log$ MAR charts. For 0.1 logMAR chart reading, previous studies reported repeatability mean sigmas of $\sim 0.04$ to $0.05 \log$ MAR ( $95 \%$ confidence limits: 0.086 to $0.100 \log$ MAR) among young subjects, and up to $0.08 \log$ MAR ( $95 \%$ confidence limits: $0.130 \log$ MAR) among older individuals for letter-by-letter scoring. Our repeatability scores using the CVA are slightly lower than that reported with ETDRS likely because our subjects were patients with clinical problems, while most ETDRS studies often tested young motivated optometry students without refractive errors. ${ }^{22,23}$ Second, the ETDRS chart test and the CVA test are designed with different size steps; on an ETDRS-style chart each letter accounts for $0.02 \log$ MAR in size, while the CVA uses 0.05 $\log$ MAR size steps. Random fluctuations in repeatability will lead to poorer repeatability of the CVA because of its coarser scale. ${ }^{2,23}$ Third, letter-by-letter scoring (as measured using the ETDRS chart) is dependent upon the termination criteria of the examiner and whether the examined individual is forced to proceed through the entire chart to the end with encouraged guessing, ${ }^{6}$ as opposed to proceeding downward to a line in which none of the letters are guessed correctly. ${ }^{24}$ If the examiner knows the prior test result, this will influence his/her termination criteria. Therefore, in a clinical setting testing of real patients, in which the examiner cannot control or influence the automated CVA test, and in which guessing is allowed but not forced, the resultant mean test-retest coefficient of repeatability is elevated to levels of 0.10 to $0.14 \log$ MAR with $95 \%$ confidence limits of 0.19 to 0.24 $\log \mathrm{MAR}$.

In conclusion, the automated CVA showed the problems that AMD patients have in a six-domain reallife vision simulation scenario and helped explaining the complaints that such individuals have under timed conditions. We suggest that testing vision using timed tests across different lighting/contrast situations will help ophthalmologists in better understanding the quality of vision in AMD patients compared to the standard ETDRS charts test.

\section{Summary}

\section{What was known before}

- Patients with AMD often complain about difficulties performing tasks of daily living. The Snellen chart test and the ETDRS chart test are the current standard methods for testing BCVA in clinical and research settings. However, these tests allow the patient to take an indefinite amount of time to recognize the target; this is not reproducing real-world experiences, which are timedependent. Moreover, these chart tests use high-contrast black letters against an unchanging white background of one luminance in the moderate photopic environment of the examination office. For these reasons, it has suggested that these chart tests are not a useful determination of vision in other contrast or luminance environments, and those measurements are poorly correlated with daily living activities that require the patient to assess visual stimuli in multiple lighting, contrast, or glare conditions against a varied background color or luminance.

\section{What this study adds}

- We used a new automated vision testing instrument, the Central Vision Analyzer, to evaluate visual function in different mesopic- and backlight-glare environments in eyes with AMD compared to normal eyes, using timedependent settings to simulate real-life conditions. We found that BCVA scores reduced significantly with decreasing contrast and luminance conditions in both normal and AMD eyes. However, the visual function in AMD eyes was more affected than in normal eyes by the decreasing contrast in mesopic conditions; that is, the magnitude of vision drop was larger for AMD eyes than for normal eyes. In addition, we found that ETDRS charts measured a better acuity compared to the corresponding module using the CVA (timed test) for AMD eyes. This means that AMD eyes have significantly lower acuity than expected under timed conditions. 


\section{Conflict of interest}

The authors declare no conflict of interest.

\section{Acknowledgements}

This study was supported by NIH grants R01EY007366 and R01EY018589 (WRF), R01EY016323 (D-UB), and in part by an unrestricted fund from Research to Prevent Blindness to the Department of Ophthalmology, University of California, San Diego.

\section{References}

1 Khandhadia S, Cherry J, Lotery AJ. Age-related macular degeneration. Adv Exp Med Biol 2012; 724: 15-36.

2 Age-Related Eye Disease Study 2 Research Group. Lutein + zeaxanthin and omega-3 fatty acids for age-related macular degeneration: the Age-Related Eye Disease Study 2 (AREDS2) randomized clinical trial. JAMA 2013; 309(19): 2005-2015.

3 Rosenfeld PJ, Brown DM, Heier JS, Boyer DS, Kaiser PK, Chung CY et al. Ranibizumab for neovascular age-related macular degeneration. $N$ Engl J Med 2006; 355(14): 1419-1431.

4 Lim LA, Frost NA, Powell RJ, Hewson P. Comparison of the ETDRS logMAR, 'compact reduced logMar' and Snellen charts in routine clinical practice. Eye (Lond) 2010; 24(4): 673-677.

5 Mitchell J, Bradley C. Quality of life in age-related macular degeneration: a review of the literature. Health Qual Life Outcomes 2006; 4: 97.

6 Ferris 3rd, FL, Kassoff A, Bresnick GH, Bailey I. New visual acuity charts for clinical research. Am J Ophthalmol 1982; 94(1): 91-96.

7 Yavuz GA, Unver YB, Bekiroglu N, Presti P, Sinclair SH. Central field perimetry of discriminated targets: I. Results for normal individuals using high-contrast targets. Eye (Lond) 2009; 23(11): 2082-2089.

8 Bradley A, Thomas T, Kalaher M, Hoerres M. Effects of spherical and astigmatic defocus on acuity and contrast sensitivity: a comparison of three clinical charts. Optom Vis Sci 1991; 68(6): 418-426.

9 Pesudovs K, Hazel CA, Doran RM, Elliott DB. The usefulness of Vistech and FACT contrast sensitivity charts for cataract and refractive surgery outcomes research. Br J Ophthalmol 2004; 88(1): 11-16.
10 Ruamviboonsuk P, Tiensuwan M, Kunawut C, Masayaanon P. Repeatability of an automated Landolt $\mathrm{C}$ test, compared with the early treatment of diabetic retinopathy study (ETDRS) chart testing. Am J Ophthalmol 2003; 136(4): 662-669.

11 Gutstein W, Sinclair SH, North RV, Bekiroglu N. Screening athletes with Down syndrome for ocular disease. Optometry 2010; 81(2): 94-99.

12 Gomez ML. Measuring the Quality of vision after cataract surgery. In "Cataract surgery and lens implantation". Curr Opin Ophthalmol 2014; 25(1): 3-11.

13 Barteselli G, Chhablani J, Gomez ML, Doede AL, Dustin L, Kozak I et al. Visual function assessment in simulated real-life situations in HIV-infected subjects. PLoS One 2014; 9(5): e97023.

14 Vos JJ. On the cause of disability glare and its dependence on glare angle, age and ocular pigmentation. Clin Exp Optom 2003; 86(6): 363-370.

15 Luo MR, Cui G, Rigg B. The development of the CIE 2000 colour-difference formula: CIEDE2000. Color Res Appl 2001; 26(5): 340-350.

16 Peli E. Contrast in complex images. J Opt Soc Am A 1990; 7(10): 2032-2040.

17 Bland JM, Altman DG. Measurement error. BMJ 1996; 312(7047): 1654.

18 Falkenstein IA, Cochran DE, Azen SP, Dustin L, Tammewar AM, Kozak I et al. Comparison of visual acuity in macular degeneration patients measured with Snellen and early treatment diabetic retinopathy study charts. Ophthalmology 2008; 115(2): 319-323.

19 Fletcher DC, Schuchard RA. Visual function in patients with choroidal neovascularization resulting from age-related macular degeneration: the importance of looking beyond visual acuity. Optom Vis Sci 2006; 83(3): 178-189.

20 Bellmann C, Unnebrink K, Rubin GS, Miller D, Holz FG. Visual acuity and contrast sensitivity in patients with neovascular age-related macular degeneration. Results from the Radiation Therapy for Age-Related Macular Degeneration (RAD-) Study. Graefes Arch Clin Exp Ophthalmol 2003; 241(12): 968-974.

21 Nguyen NX, Weismann M, Trauzettel-Klosinski S. Improvement of reading speed after providing of low vision aids in patients with age-related macular degeneration. Acta Ophthalmol 2009; 87(8): 849-853.

22 Raasch TW, Bailey IL, Bullimore MA. Repeatability of visual acuity measurement. Optom Vis Sci 1998; 75(5): 342-348.

23 Arditi A, Cagenello R. On the statistical reliability of letter-chart visual acuity measurements. Invest Ophthalmol Vis Sci 1993; 34(1): 120-129.

24 Bailey IL, Lovie JE. New design principles for visual acuity letter charts. Am J Optom Physiol Opt 1976; 53(11): 740-745. 